\title{
Z

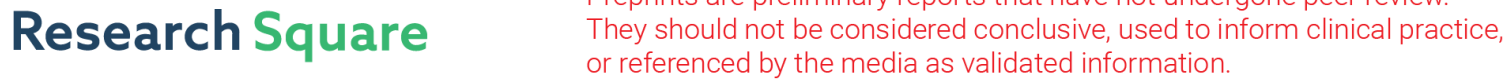 \\ Identification of Potential Autophagy-Related Genes in Steroid-Induced Osteonecrosis of the Femoral Head
}

\section{XueZhen LIANG}

Shandong University of Traditional Chinese Medicine Affiliated Hospital https://orcid.org/0000-00015649-4212

\section{Di LUO}

Shandong University of Traditional Chinese Medicine Affiliated Hospital

\section{Yan-Rong CHEN}

Shandong University of Traditional Chinese Medicine Affiliated Hospital

\section{Jia-Cheng LI}

Shandong University of Traditional Chinese Medicine

\section{Bo-Zhao YAN}

Shandong University of Traditional Chinese Medicine

\section{Yan-Bo GUO}

Shandong University of Traditional Chinese Medicine

\section{Ming-Tao WEN}

Shandong University of Traditional Chinese Medicine

\section{Bo XU}

Shandong University of Traditional Chinese Medicine

Gang LI ( $\nabla$ sdszylg@163.com )

Affiliated Hospital of Shandong University of Traditional Chinese Medicine https://orcid.org/00000002-4587-6650

\section{Research article}

Keywords: steroid induced osteonecrosis of the femoral head, autophagy, bioinformatics analysis, gene expression omnibus

Posted Date: November 15th, 2021

DOI: https://doi.org/10.21203/rs.3.rs-1066425/v1

License: (c) (1) This work is licensed under a Creative Commons Attribution 4.0 International License. Read Full License 


\section{Abstract}

Purpose: Steroid-induced osteonecrosis of the femoral head (SONFH) was a refractory orthopedic hip joint disease in the young and middle-aged people. Previous experimental studies had shown that autophagy might be involved in the pathological process of SONFH, but the pathogenesis of autophagy in SONFH remained unclear. We aim to identify and validate the key potential autophagy-related genes of SONFH to further illustrate the mechanism of autophagy in SONFH through bioinformatics analysis.

Methods: The mRNA expression profile dataset GSE123568 was download from Gene Expression Omnibus (GEO) database, including 10 non-SONFH (following steroid administration) samples and 30 SONFH samples. The autophagy-related genes were obtained from the Human Autophagy Database (HADb). The autophagy-related genes of SONFH were screened by intersecting GSE123568 dataset with autophagy genes. The differentially expressed autophagy-related genes of SONFH were identified by $\mathrm{R}$ software. Besides, the Gene Ontology (GO) enrichment analysis and Kyoto Encyclopedia of Genes and Genomes (KEGG) pathway enrichment analysis was conducted for the differentially expressed autophagy-related genes of SONFH by R software. Then, the correlation analysis between the expression levels of differentially expressed autophagy-related genes of SONFH was confirmed by R software. Moreover, the protein-protein interaction (PPI) network were analyzed by the Search Tool for the Retrieval of Interacting Genes (STRING), and the significant gene cluster modules were identified by the MCODE Cytoscape plugin, and hub genes of differentially expressed autophagy-related genes of SONFH were screened by the CytoHubba Cytoscape plugin. Finally, the expression levels of hub genes of differentially expressed autophagy-related genes of SONFH was validated in hip articular cartilage specimens from necrosis femur head (NFH) by GSE74089 dataset.

Results: A total of 34 differentially expressed autophagy-related genes were identified between the peripheral blood of SONFH samples and non-SONFH Samples based on the defined criteria, including 25 up-regulated genes and 9 down-regulated genes. The GO and KEGG pathway enrichment analysis revealed that these 34 differentially expressed autophagy-related genes of SONFH were concentrated in death domain receptors, FOXO signaling pathway and apoptosis. The correlation analysis revealed a significant correlation among the 34 differentially expressed autophagy-related genes of SONFH. The PPI results demonstrated that the 34 differentially expressed autophagy-related genes interacted with each other. There were 10 hub genes identified by the MCC algorithms of Cytohubba. The results of GSE74089 dataset showed TNFSF10, PTEN and CFLAR were significantly upregulated while BCL2L1 were significantly downregulated in the hip cartilage specimens, which were consistent with the GSE123568 dataset.

Conclusions: There were 34 potential autophagy-related genes of SONFH identified using bioinformatics analysis. TNFSF10, PTEN, CFLAR and BCL2L1 might serve as potential drug targets and biomarkers by regulating autophagy. These results would expand new insights into the autophagy-related understanding of SONFH and might be useful in the diagnosis and prognosis of SONFH. 


\section{Introduction}

Steroid induced osteonecrosis of the femoral head (SONFH) was a chronic refractory orthopedic disease characterized by accumulation of microfractures without continuous remodeling or the collapse of the femoral head, with a high mortality, disability and dysfunction[1, 2]. SONFH occurred most frequently in young and middle-aged adults, and if without effective systematic treatment, about $80 \%$ of SONFH patients would develop to femoral head collapse within 1 4 years, and then required artificial joint replacement [3]. Although glucocorticoids (GC) had been recognized as a risk factor for SONFH, its exact pathogenesis had not been well defined. As the SONFH study deepened, accumulating highly recognized mechanism hypotheses of pathogenicity had been proposed to explain their developmental mechanisms, including uncoupled bone remodeling or unbalanced osteogenic and adipogenic differentiation of BMSCs, cell proliferation, programmed cell death [4]. Among these biological processes, programmed cell death played a critical role in the development of SONFH. Autophagy, a programmed cell death-like process, has been identified after SONFH, but the mechanism of autophagy in bone repair and bone homeostasis remained unclear.

Autophagy was an evolutionarily conserved intracellular catabolic process that contributed to degrade accumulated and unnecessary intracellular materials in lysosomes, which differed from apoptosis[5]. The discovery of autophagy mechanisms was recognized by the 2016 Nobel Prize in Physiology or Medicine[6]. Recent studies had revealed that autophagy was related to the various diseases including SONFH, and the expression levels of some key targets changes after autophagy[7-10]. For instance, as the proteins necessary for autophagy, Atg5, Atg7, Atg4B, and LC3, played a key role in the production of osteoclast crinkle boundaries and the secretory function of osteoclasts and in vitro and in vivo maintaining balance and homeostasis[11]. Moreover, FIP200 was an integral part of the ULKs-Atg13FIP200 complex and regulated autophagy in osteoblasts that affected bone fragility and bone loss[12]. In addition, it had been reported that several signaling pathways influenced the biological function of SONFH through autophagy. The mTOR pathway was the main regulation of mammalian autophagy, estradiol had recently been found to increase the level of autophagy through ER-ERK-mTOR pathway to prevent osteoblast apoptosis in SONFH[13]. However, many autophagy-related genes of SONFH had not yet been found, so further studies of autophagy-related genes of SONFH genes needed to be explored.

At present, there are no bioinformatics-based studies on the pathogenesis of autophagy-related genes after SONFH. Zhang Y completed a SONFH-related dataset GSE123568 with genome-wide of Human peripheral serum, which contained normal patient serum samples and SONFH serum samples[14]. We could use machine learning algorithms and multiple bioinformatic approaches to screen and determine the differentially expressed genes between SONFH patients and normal individuals based on the microarray dataset. In this study, we intersected these differentially expressed genes with the ferroptosis dataset to obtain differentially expressed autophagy-related genes. Then, the Gene Ontology (GO) and the Kyoto Encyclopedia of Genes and Genomes (KEGG) was use for enrichment analysis of the differentially expressed autophagy-related genes to establish the pathogenesis of SONFH. Moreover, the correlation analysis was to explore the association of the identified differentially expressed autophagy-related genes 
with each other. Furthermore, we conducted the protein-protein interactions (PPI) network to identify hub genes. Finally, the expression levels of crucial differentially expressed autophagy-related genes were further verified in GSE74089 dataset with hip cartilage specimens. Therefore, our results would reveal the potential autophagy-related genes of SONFH, and help to understand the pathogenesis of autophagy in SONFH, and provide potential biomarkers for clinical diagnosis and treatment of SONFH.

\section{Materials And Methods}

\subsection{Autophagy-Related Genes Datasets and Microarray Data}

The Human Autophagy Database (HADb) was the first Human Autophagy-dedicated Database, which was a public repository containing information about the human genes described so far as involved in autophagy (http://www.autophagy.lu/index.html) [15].

The mRNA expression profile dataset of GSE123568 was obtained from National Center for Biotechnology Information Gene Expression Omnibus (NCBI GEO, https ://www.ncbi.nlm.nih.gov/geo/) database according to the following inclusion criteria: (1) "Steroid induced Osteonecrosis of the Femoral Head" or "SONFH"; (2) Homo sapiens; (3) "Expression Profiling by array" or "Expression profiling by high throughput sequencing". The GSE123568 was submitted on Dec 10, 2018 and updated on Jan 01, 2020 by Zhang et al and included 10 non-SONFH (following steroid administration) samples and $30 \mathrm{SONFH}$ samples(https://www.ncbi.nlm.nih.gov/geo/query/acc.cgi?acc=GSE123568), and the platform of the dataset was GPL15207 [PrimeView] Affymetrix Human Gene Expression Array.

\subsection{Differentially Expressed Analysis of Autophagy-Related Genes in SONFH}

To pre-process and normalize the original files of GSE123568, we downloaded the CEL files of raw data of GSE123568, and used the "affy" R package based on Robust Multiarray Average (RMA) method. To observe the overall distribution in non-SONFH samples and SONFH samples and identify the presence of singular samples, we projected the high-dimensional repeatability of data in GSE123568 into twodimensional space for principal component analysis (PCA). To identify the differentially expressed autophagy-related genes in SONFH, we used "limma" R package, and an adjusted P-value $<0.05$ and $\| \log _{2}$ fold change $(\mathrm{FC}) \mid>\log _{2} 1.5$ were considered as the screening criteria of differentially expressed genes. The PCA, heatmaps and volcano plots were computed and visualized by "PCA", "pheatmap" and "ggplot2" R package.

\subsection{Functional Enrichment Analysis of Autophagy-Related Genes in SONFH}

To analyze the identified differentially expressed autophagy-related genes in SONFH, the GO and the KEGG pathway enrichment analysis was conducted and visualized in R software using "clusterProfiler" and "GOplot" R package. The GO enrichment analysis mainly consisted of biological process (BP), 
cellular composition (CC), and molecular function (MF). An adjusted $p$ value $<0.05$ was considered as the screening criteria for significantly enriched terms.

\subsection{Correlation Analysis of the Differentially Expressed Autophagy-Related Genes in SONFH}

To confirm the correlation between the expression levels of differentially expressed autophagy-related genes in SONFH, we used Spearman correlation coefficient in the "corrplot" R package.

\subsection{Construction of PPI network and Screening of Hub Genes and Key Modules}

To gain more insight into the in-depth relationships of differentially expressed autophagy-related genes in SONFH, we analyzed and constructed the PPI network using the Search Tool for the Retrieval of Interacting Genes (STRING, Version: 11.0, https://string-db.org/) and Cytoscape software (version: 3.7.2, http://cyto-scape.org/). The thresholds for a combined score was set to medium confidence $>=0.4$, and the isolated nodes were discarded. We used molecular complex detection (MCODE) plugin to identify clustering analysis of gene networks for selecting the key sub-network modules. We explored important nodes/hubs and fragile motifs in an interactome network by using CytoHubb plugin, which provided 11 topological analysis algorithms including Degree, Edge Percolated Component (EPC), Maximum Neighborhood Component (MNC), Density of Maximum Neighborhood Component (DMNC), Maximal Clique Centrality (MCC) and centralities based on shortest paths, such as Bottleneck (BN), EcCentricity, Closeness, Radiality, Betweenness, and Stress.

\subsection{Cross-Validation of External Dataset for the differentially expressed autophagy-related hub genes in SONFH}

The mRNA expression dataset GSE74089 associated with necrosis femur head (NFH) was downloaded from the GEO database, which included 4 hip cartilage samples from patients with NFH and 4 hip cartilage samples from healthy controls. And the platform of the dataset was GPL13497 Agilent-026652 Whole Human Genome Microarray 4x44K v2. To achieve the cross-validation, we analyzed the expression levels of differentially expressed autophagy-related hub genes in GSE74089 dataset, and compared them with the expression levels in GSE123568 dataset in the study.

\subsection{Statistical Analysis}

The data statistical analysis was performed using R software (version 4.0.2) in this study. Gene expression levels of samples in GSE123568 and GSE74089 were compared using Kruskal-Wallis test. $P<0.05$ was set as a threshold for significant statistical significance

\section{Results}




\subsection{Differentially Expressed Autophagy-Related Genes in SONFH-Retrospective Analysis of Autophagy-Related Genes}

To confirmed the cluster analysis data and evaluate the repeatability of GSE123568, we subjected the data to PCA, and the results showed that the repeatability of data in GSE74089 was considered reliable in Figure 1A. We next analyzed the expression of 222 autophagy-related genes of HADb in 30 SONFH patients and 10 non-SONFH patients (following steroid administration), and 34 differentially expressed autophagy-related genes were identified based on the defined criteria of an adjusted P-value $<0.05$ and $|\log F C|>\log _{2} 1.5$, including 25 up-regulated genes and 9 down-regulated genes in SONFH samples compared to non-SONFH Samples in Table 1.

A total of 222 non-duplicated genes were excavated from the HADb and intersected them with GSE123568 to identify differentially expressed autophagy-related genes. The 34 differentially expressed autophagy-related genes of GSE123568 were shown in the volcano plots and the hierarchical clustering heatmap by the ggplot and pheatmap package in the R software in Figure 1B and Figure 1C. Immediately after, the expression patterns of 34 differentially expressed autophagy-related genes between SONFH and non-SONFH samples were shown in box plot in Figure 2. 
Table 1

the 34 differentially expressed autophagy-related genes in SONFH samples compared to healthy samples

\begin{tabular}{|c|c|c|c|c|c|}
\hline $\begin{array}{l}\text { Gene } \\
\text { ID }\end{array}$ & Name & Symbol & $\log F C$ & P.Value & adj.P.Val \\
\hline 729230 & chemokine (C-C motif) receptor 2 & CCR2 & 1.357 & $2.18 \mathrm{E}-05$ & 0.000425 \\
\hline 8743 & $\begin{array}{l}\text { tumor necrosis factor (ligand) } \\
\text { superfamily, member } 10\end{array}$ & TNFSF10 & 1.157 & 0.000195 & 0.002057 \\
\hline 4179 & $\begin{array}{l}\text { CD46 molecule, complement } \\
\text { regulatory protein }\end{array}$ & CD46 & 1.122 & $1.84 \mathrm{E}-05$ & 0.000378 \\
\hline 5265 & $\begin{array}{l}\text { serpin peptidase inhibitor, clade } A \\
\text { (alpha-1 antiproteinase, antitrypsin), } \\
\text { member } 1\end{array}$ & SERPINA1 & 1.091 & 2.83E-06 & 9.69E-05 \\
\hline 3920 & $\begin{array}{l}\text { lysosomal-associated membrane } \\
\text { protein } 2\end{array}$ & LAMP2 & 0.998 & $2.15 \mathrm{E}-05$ & 0.00042 \\
\hline 5580 & protein kinase $\mathrm{C}$, delta & PRKCD & 0.988 & $6.25 \mathrm{E}-06$ & 0.000173 \\
\hline 834 & $\begin{array}{l}\text { caspase 1, apoptosis-related cysteine } \\
\text { peptidase (interleukin 1, beta, } \\
\text { convertase) }\end{array}$ & CASP1 & 0.972 & $6.74 \mathrm{E}-05$ & 0.000934 \\
\hline 89849 & $\begin{array}{l}\text { ATG } 16 \text { autophagy related } 16 \text {-like } 2 \text { (S. } \\
\text { cerevisiae) }\end{array}$ & ATG16L2 & 0.928 & $2.4 \mathrm{E}-07$ & $1.71 \mathrm{E}-05$ \\
\hline 10135 & $\begin{array}{l}\text { nicotinamide } \\
\text { phosphoribosyltransferase }\end{array}$ & NAMPT & 0.897 & 0.000113 & 0.001367 \\
\hline 637 & $\mathrm{BH} 3$ interacting domain death agonist & BID & 0.886 & 4.81E-07 & $2.88 \mathrm{E}-05$ \\
\hline 5894 & $\begin{array}{l}\text { v-raf-1 murine leukemia viral oncogene } \\
\text { homolog } 1\end{array}$ & RAF1 & 0.872 & 5.37E-06 & 0.000155 \\
\hline 23001 & $\begin{array}{l}\text { WD repeat and FYVE domain } \\
\text { containing } 3\end{array}$ & WDFY3 & 0.848 & 0.000309 & 0.002868 \\
\hline 837 & $\begin{array}{l}\text { caspase } 4 \text {, apoptosis-related cysteine } \\
\text { peptidase }\end{array}$ & CASP4 & 0.825 & 0.000148 & 0.001672 \\
\hline 10287 & regulator of G-protein signaling 19 & RGS19 & 0.821 & 1.83E-05 & 0.000377 \\
\hline 8837 & $\begin{array}{l}\text { CASP8 and FADD-like apoptosis } \\
\text { regulator }\end{array}$ & CFLAR & 0.821 & 5.42E-05 & 0.000803 \\
\hline 355 & $\begin{array}{l}\text { Fas (TNF receptor superfamily, } \\
\text { member 6) }\end{array}$ & FAS & 0.784 & 0.000757 & 0.005384 \\
\hline 8682 & $\begin{array}{l}\text { phosphoprotein enriched in astrocytes } \\
15\end{array}$ & PEA15 & 0.761 & $2.75 \mathrm{E}-05$ & 0.000493 \\
\hline 7852 & chemokine (C-X-C motif) receptor 4 & CXCR4 & 0.747 & 0.000432 & 0.003674 \\
\hline
\end{tabular}




\begin{tabular}{|c|c|c|c|c|c|}
\hline $\begin{array}{l}\text { Gene } \\
\text { ID }\end{array}$ & Name & Symbol & $\log \mathrm{FC}$ & P.Value & adj.P.Val \\
\hline 9341 & $\begin{array}{l}\text { vesicle-associated membrane protein } 3 \\
\text { (cellubrevin) }\end{array}$ & VAMP3 & 0.724 & $7.26 \mathrm{E}-05$ & 0.000989 \\
\hline 1027 & $\begin{array}{l}\text { cyclin-dependent kinase inhibitor 1B } \\
\text { (p27, Kip1) }\end{array}$ & CDKN1B & 0.723 & $1.32 \mathrm{E}-05$ & 0.000296 \\
\hline 5573 & $\begin{array}{l}\text { protein kinase, cAMP-dependent, } \\
\text { regulatory, type I, alpha (tissue specific } \\
\text { extinguisher 1) }\end{array}$ & PRKAR1A & 0.693 & 5.49E-05 & 0.000809 \\
\hline 5728 & phosphatase and tensin homolog & PTEN & 0.666 & 0.000695 & 0.0051 \\
\hline 824 & calpain 2, (m/II) large subunit & CAPN2 & 0.626 & 0.000103 & 0.001271 \\
\hline 5595 & mitogen-activated protein kinase 3 & MAPK3 & 0.625 & 3.48E-05 & 0.000581 \\
\hline 3916 & $\begin{array}{l}\text { lysosomal-associated membrane } \\
\text { protein } 1\end{array}$ & LAMP1 & 0.608 & $6.33 \mathrm{E}-06$ & 0.000174 \\
\hline 81631 & $\begin{array}{l}\text { microtubule-associated protein } 1 \text { light } \\
\text { chain } 3 \text { beta }\end{array}$ & MAP1LC3B & -0.720 & $2.55 \mathrm{E}-06$ & 8.96E-05 \\
\hline 6767 & $\begin{array}{l}\text { suppression of tumorigenicity } 13 \\
\text { (colon carcinoma) (Hsp70 interacting } \\
\text { protein) }\end{array}$ & ST13 & -0.954 & 2.3E-09 & $6.76 \mathrm{E}-07$ \\
\hline 65018 & PTEN induced putative kinase 1 & PINK1 & -0.976 & $1.53 \mathrm{E}-05$ & 0.00033 \\
\hline 6794 & serine/threonine kinase 11 & STK11 & -1.097 & 2.19E-10 & 1.11E-07 \\
\hline 11345 & $\begin{array}{l}\text { GABA(A) receptor-associated protein- } \\
\text { like } 2\end{array}$ & GABARAPL2 & -1.108 & $2.6 \mathrm{E}-09$ & 7.1E-07 \\
\hline 2281 & FK506 binding protein 1B, $12.6 \mathrm{kDa}$ & FKBP1B & -1.159 & $2.21 \mathrm{E}-05$ & 0.000427 \\
\hline 598 & BCL2-like 1 & BCL2L1 & -1.197 & 8.34E-07 & 4.12E-05 \\
\hline 2309 & forkhead box 03 & F0X03 & -1.221 & $2.62 \mathrm{E}-07$ & $1.82 \mathrm{E}-05$ \\
\hline 665 & $\begin{array}{l}\text { BCL2/adenovirus E1B 19kDa } \\
\text { interacting protein 3-like }\end{array}$ & BNIP3L & -1.412 & $6.44 \mathrm{E}-08$ & 6.77E-06 \\
\hline
\end{tabular}

\subsection{GO and KEGG Enrichment Analysis of the Differentially Expressed Autophagy-Related Genes}

To analyze the potential biological process and KEGG pathway of these 34 differentially expressed autophagy-related genes, we conducted GO and KEGG enrichment analysis by using clusterProfiler R package. There were 415 significant-enriched $\mathrm{GO}$ biological process terms, and the results involved in extrinsic apoptotic signaling pathway via death domain receptors, regulation of extrinsic apoptotic signaling pathway via death domain receptors, autophagy, process utilizing autophagic mechanism and 
response to oxygen levels, which were shown in Figure 3A and Figure 4A and Figure 4B. In KEGG enrichment analysis, the 34 differentially expressed autophagy-related genes were significantly enriched in 69 KEGG pathway terms, such as autophagy, Foxo signaling pathway, apoptosis, mitophagy, NOD-like receptor signaling pathway in Figure 3D and Figure 4C and Figure 4D

\subsection{Correlation Analysis of the Differentially Expressed Autophagy-Related Genes}

We performed the correlation analysis in order to explore the expression correlation among these 34 autophagy-related genes in GSE123568 dataset, and the results shown in Figure 5. The results suggested that part of these autophagy -related genes showed weak-to-moderate correlation. In these 34 genes, RAF1 and ATG16L2 were most positively correlated (Cor=0.95). Nevertheless, there were also some negatively correlated genes in these 34 genes, including ST13 and CFLAR (Cor=-0.86).

\subsection{PPI Network and Screening of Key Modules and Hub Genes}

To determine the in-depth interactions among the 34 differentially expressed autophagy-related genes, PPI analysis were performed using the STRING database. These autophagy-related genes interacted with each other and the interaction number of each gene were visualized by Cytoscape in Figure 6A. To identify the significant gene cluster modules, we used MCODE plugin. The results showed 3 clusters in Figure 6B-D. Cluster 1 had the highest cluster score that was comprised of 10 nodes and 28 edges, which were 4 up-regulated and 6 down-regulated differentially expressed genes (score: 6.222), followed by cluster 2 and cluster 3 that were both comprised of 3 nodes and 3 edges (score: 3.000). Meanwhile, to identify hub genes, the CytoHubba plugin was used. The results demonstrated that 10 hub genes were identified by the MCC algorithms of Cytohubba, including TNFSF10, BCL2L1, PINK1, MAP1LC3B, BNIP3L, GABARAPL2, CFLAR, PTEN, FOX03, and MAPK3.

\subsection{Validation the Differentially Expressed Autophagy- Related Genes in GSE74089 dataset}

To validate the reliability of the GSE123568 dataset, the expression levels of 10 hub genes of differentially expressed autophagy-related genes were further identified by GSE74089 dataset. The microarray dataset GSE74089 about identification of potential biomarkers in hip articular cartilage specimens of NFH were downloaded from GEO database, which included the 4 NFH patients and 4 healthy controls. Similar to the results of mRNA microarray in blood samples, the expression levels of TNFSF10, PTEN and CFLAR were significantly higher, and the expression levels of BCL2L1 were significantly decreased in hip articular cartilage specimens of SONFH samples than in hip articular cartilage specimens of normal samples, which might play an important role in autophagy after steroid administration and shown in Figure 7. However, the expression levels of MAPK3, MAP1LC3B, GABARAPL2, FOX03, and BNIP3L showed significant difference between the two groups, but contrary to expression in the GSE123568 dataset, and PINK1 had no expression level in GSE74089 dataset. 


\section{Discussion}

SONFH, which represented a common, progressive and refractory orthopedics disease, was one of the most frequent cause of hip disability worldwide [16]. The main manifestation of the SONFH in the early stage was the pathological change of bone trabecular combined with a lack of adequate replacement of the cells, and its clinical symptoms were not obvious. With the progress of SONFH, the patient's femoral head appeared local sclerosis and cystic change, even collapse, which seriously affected the normal life and work of the patient [17]. The exact pathogenesis had not been defined, but with studies on femoral head necrosis deepening, many highly recognized hypotheses of pathogenicity had been proposed to explain the mechanism of SONFH, such as the programmed cell death[18-20]. The programmed cell death was an active process that regulated the development of the body and maintained a stable internal environment in multicellular organisms, which was controlled by the relevant genes [21]. It was well known that autophagy was the key common form of programmed cell death in organisms, which regulated negative effects of GC on the osteoblasts and osteoclast and osteocytes[22]. However, the potential biomarkers and regulatory mechanisms of autophagy in the pathological process of SONFH remained unclear. Therefore, the study explored several genes that had not yet been mentioned in the area of autophagy and SONFH, which would provide effective reference and have important guiding significance for the clinical diagnosis and treatment of SONFH patients.

To the best of our knowledge, several published articles related to bone metabolic diseases have explored autophagy-related genes. A review about the role of autophagy in bone homeostasis and the onset of osteoporosis introduced the relevance of autophagy in bone physiology, and discussed its role and therapeutic potential of autophagy in the pathogenesis of osteoporosis[23]. However, the bioinformatics analysis of autophagy-related genes has not been explored in SONFH. In the study, we obtained the first identified 34 potential differentially expressed autophagy-related genes in SONFH from the intersection of differentially expressed genes of the datasets GSE123568 and autophagy-related of HADb using bioinformatics analysis, including 25 up-regulated genes and 9 down-regulated genes. Some of these differentially expressed autophagy-related genes of SONFH had been previously studied. For example, Ye Yaping et al demonstrated that the phosphatase and tensin homolog (PTEN), a tumor suppressor gene that promoted cell apoptosis, was found to be elevated in SONFH patients [24]. In addition, accumulating evidence suggested that FAS mRNA expression levels in MC3T3-E1 cells correlate to bone or osteoblast cell apoptosis in SONFH patients[25]. More future studies were needed to be performed to confirm potential differentially expressed autophagy-related genes of SONFH.

To clarify the underlying molecular mechanisms of the 34 differentially expressed autophagy-related genes of SONFH, we conducted biological functions through GO and KEGG enrichment analysis. The GO and KEGG enrichment analysis revealed that the differentially expressed autophagy-related genes of SONFH were significantly concentrated in several enriched terms related to autophagy, apoptosis, mitophagy, FOXO signaling pathway and NOD-like receptor signaling pathway. Previous studies of the FOXO signaling pathway had focused on the regulation of bone cell homeostasis via FOXO induction effect on autophagy[26]. A previous bioinformatics-based study demonstrated that toll-like receptor 
(TLR), neurotrophin and NOD-like receptor signaling pathway were most likely to be regulated by the differential miRNAs involved in SONFH[27]. Another recent study showed that GC could induce autophagy and apoptosis of bone cells and was related to the dose of GC. In other words, apoptosis was activated by high dose of GC, while low dose of GC causes autophagy[28]. Clearly, there were still many more studies to corroborate our results and gain more insights in the potential biological functions of these differentially expressed autophagy-related genes of SONFH.

Afterwards, the PPI network of differentially expressed autophagy-related genes of SONFH was constructed by the STRING database, and the node topological characteristics and inter-node interaction were analyzed to reveal the biological mechanisms related to the PPI network by the NetworkAnalyzer plug-in of Cytoscape. Biological networks might consist of several functional modules, where the complex subunits and their interactions often leaded to the same biological processes, providing a new perspective into the biological functions that constituted the various components of the complex network. The results showed 3 cluster modules from the PPI network were extracted by MCODE analysis. Cluster 1 had the highest cluster score that was comprised of 10 nodes and 28 edges, which were 4 upregulated and 6 down-regulated differentially expressed genes including PINK1, BCL2L1, BNIP3L, MAP1LC3B, GABARAPL2, CASP1, PTEN, FAS, BID and FOX03. The filtered hub nodes also varied with different filtering criteria. Furthermore, the top10 hub genes involved in autophagy were identified by the MCC algorithms of Cytohubba with the reference to Gov E, including TNFSF10, BCL2L1, PINK1, MAP1LC3B, BNIP3L, GABARAPL2, CFLAR, PTEN, FOXO3, and MAPK3[29]. All 10 hub genes identified from Cytohubba were present in the intersection of Cytohubba and 3 cluster modules.

Based on bioinformatics analysis results of GSE123568 dataset in blood samples, the expression levels of top 10 hub differentially expressed autophagy-related genes were further identified by GSE74089 dataset in hip articular cartilage specimens. The TNFSF10, PTEN and CFLAR were significantly upregulated while BCL2L1 were significantly downregulated, which were consistent both in the GSE123568 dataset and GSE123568 dataset. Several studies on these consistently expressed genes had been reported to be associated with bone metabolic diseases or autophagy. For instance, Komori et al indicated that BCL2L1 overexpression in osteoblasts increased the trabecular and cortical bone volumes with normal structures and maintained them majorly by preventing osteoblast apoptosis in osteoporosis [30]. Liu and his colleagues put forward that TNFSF10 overexpression probably stimulated proliferation and inflammation, and inhibited apoptosis by regulating the miR-376-3p/FGFR1 axis in osteoarthritis[31]. $\mathrm{Xu}$ et al demonstrated PTEN were found to affect the risk of osteoarthritis development by regulating expression of autophagy regulators and the MTOR pathway[32]. However, there were still few related studies on the consistently expressed genes mentioned in the context of autophagy and SONFH. These novel genes provide new concepts in autophag after SONFH, and further investigations will be intended to better understand this field.

Despite potential autophagy-related genes of SONFH were identified based on bioinformatics there were still some limitations in this study. First, although the GEO database stored large amounts of highthroughput data, we didn't find more underutilized datasets of SONFH, resulting in particularly limited 
number of samples in this study and not validated with blood samples. Second, further in vitro and in vivo experiments to validate the differentially expressed autophagy-related genes and their potential mechanisms were lacking. Therefore, further research needs to be warranted to reduce the possible limitations in terms of biased results and conclusions.

\section{Conclusion}

In conclusion, we identified 34 potential autophagy-related genes of SONFH with the help of bioinformatic analysis toolkit in the present study. Moreover, the hub consistently expressed genes TNFSF10, PTEN, CFLAR and BCL2L1 might serve as potential therapeutic targets and biomarkers for autophagy after SONFH. These results facilitated indepth exploration of the development of SONFH by regulating autophagy and provided novel insight into the diagnosis and prognosis of SONFH.

\section{Abbreviations}

biological process (BP) ; Bottleneck (BN); cellular composition (CC); Density of Maximum Neighborhood Component (DMNC); Edge Percolated Component (EPC); fold change (FC); glucocorticoids (GC); Gene Expression Omnibus (GEO); Gene Ontology (GO); Human Autophagy Database (HADb); Kyoto Encyclopedia of Genes and Genomes (KEGG); molecular complex detection (MCODE); Maximal Clique Centrality (MCC); molecular function (MF); Maximum Neighborhood Component (MNC); National Center for Biotechnology Information (NCBI); necrosis femur head (NFH); principal component analysis (PCA); protein-protein interactions (PPI); phosphatase and tensin homolog (PTEN); Robust Multiarray Average (RMA); Steroid-induced osteonecrosis of the femoral head (SONFH); Search Tool for the Retrieval of Interacting Genes (STRING); toll-like receptor (TLR).

\section{Declarations}

\section{Data Availability Statement}

Publicly available datasets were analyzed in this study. This data can be found at the following URL: HADb (http://www.autophagy.lu/index.html) and GSE123568 dataset (https://www.ncbi.nlm.nih.gov/geo/query/acc.cgi?acc=GSE123568) and GSE74089 dataset (https://www.ncbi.nlm.nih.gov/geo/query/acc.cgi?acc=GSE74089)

\section{Ethics Approval and Informed Consent}

The study was conducted in agreement with the Declaration of Helsinki and its later amendments or comparable ethical standards. Written informed consent was obtained from all individual participants included in the study. 


\section{Consent for Publication}

All participating authors give their consent for this work to be published.

\section{Acknowledgments}

We would like acknowledge the following financial support: National Natural Science Foundation of China (No. 81774333 and No. 82074453); and the Development Plan of Shandong Medical and Health Technology (No. 2019WS577); and the Development Plan of Shandong Traditional Chinese Medicine Science and Technology (No. 2020Q009); and the Innovation Program of Jinan Clinical Medicine Science and Technology (No.202019056). And we thank the NCBI GEO database for sharing the data.

\section{Funding}

This work was supported by grants from the National Natural Science Foundation of China (No. 81774333 and No. 82074453); and the Development Plan of Shandong Medical and Health Technology (No. 2019WS577); and the Development Plan of Shandong Traditional Chinese Medicine Science and Technology (No. 2020Q009); and the Innovation Program of Jinan Clinical Medicine Science and Technology (No.202019056).

\section{Author Contributions}

All authors made a significant contribution to the work reported and agreed to be accountable for all aspects of the work. L.G and L.X.Z designed the experiments. L.X.Z, L.D, L.J.C, Y.B.Z, and W.M.T performed the experiments. L.X.Z, C.Y.R, G.Y.B, and X.B prepared the initial draft of the manuscript. L.J.C, C.Y.R, X.B and L.G gave critical feedback during the study or during the submission of the manuscript. All authors had given final approval of the version to be submitted and agreed on the journal to be published.

\section{Declaration of interest}

The authors declare no conflicts of interest in the research.

\section{References}

1. Jean-Philippe H, Michel M, Viviane dM (2016) A prospective cohort study of the clinical presentation of non-traumatic osteonecrosis of the femoral head: spine and knee symptoms as clinical presentation of hip osteonecrosis. International orthopaedics 40

2. Qing-Yu Z, Zi-Rong L, Fu-Qiang G, Wei S (2018) Pericollapse Stage of Osteonecrosis of the Femoral Head: A Last Chance for Joint Preservation. Chinese medical journal 131 
3. W-L G, Q L, X W, Y W, J P, S-B L (2015) Key pathway to prevent the collapse of femoral head in osteonecrosis. European review for medical and pharmacological sciences 19

4. Luo P, Gao F, Han J, Sun W, Li Z (2018) The role of autophagy in steroid necrosis of the femoral head: a comprehensive research review. International Orthopaedics 42

5. C TA, Vanessa G, Julien P (2020) Current Evidence on Cell Death in Preterm Brain Injury in Human and Preclinical Models. Frontiers in cell and developmental biology 8

6. Levine B, Kroemer G (2019) Biological Functions of Autophagy Genes: A Disease Perspective. Cell 176

7. Liu, Wanlin, Zhao, Zhenqun, Yuyan, Meng, Chenyang, Wang, Jianzhong, Bai (2018) Dexamethasoneinduced production of reactive oxygen species promotes apoptosis via endoplasmic reticulum stress and autophagy in MC3T3-E1 cells. International Journal of Molecular Medicine

8. MD ZqZ, MD WIL, MD SbG, MD RB, MD JIY (2020) Mechanism of Methylprednisolone-Induced Primary Cilia Formation Disorder and Autophagy in Osteoblasts. Orthopaedic Surgery 12

9. Puji P, Zhigang N, Fei S, Hao P (2020) Glucocorticoids induce femoral head necrosis in rats through the ROS/JNK/c-Jun pathway. FEBS Open Bio 11

10. Wang X-Y, Gong L-J, Huang J-M, Jiang C, Yan Z-Q (2020) Pinocembrin alleviates glucocorticoidinduced apoptosis by activating autophagy via suppressing the PI3K/Akt/mTOR pathway in osteocytes. European Journal of Pharmacology 880

11. J DC, C MB, Wei Z, L BW, Eline vM, Yoshifumi T, Judith K, A TS, L TS, W VH (2011) Autophagy proteins regulate the secretory component of osteoclastic bone resorption. Developmental cell 21

12. Liu F, Fang F, Yuan H, Yang D, Chen Y, Williams L, Goldstein SA, Krebsbach PH, Guan JL (2013) Suppression of autophagy by FIP200 deletion leads to osteopenia in mice through the inhibition of osteoblast terminal differentiation. Journal of Bone and Mineral Research 28

13. Yang Y-H, Chen K, Li B, Chen J-W, Zheng X-F, Wang Y-R, Jiang S-D, Jiang L-S (2013) Estradiol inhibits osteoblast apoptosis via promotion of autophagy through the ER-ERK-mTOR pathway. Apoptosis 18

14. Li T, Zhang Y, Wang R, Xue Z, Li S, Cao Y, Liu D, Niu Y, Mao X, Wang X, Li W, Guo Q, Guo M, Lin N, Chen W (2019) Discovery and validation an eight-biomarker serum gene signature for the diagnosis of steroid-induced osteonecrosis of the femoral head. Bone 122

15. Moussay E, Kaoma T, Baginska J, Muller A, Moer KV, Nicot N, Nazarov PV, Vallar L, Chouaib S, Berchem G (2011) The acquisition of resistance to TNFa in breast cancer cells is associated with constitutive activation of autophagy as revealed by a transcriptome analysis using a custom microarray. Autophagy 7:760-770

16. De-Wei Z, Mang Y, Kai H, Wei W, Lei Y, Ben-Jie W, Xiao-Hong G, Yong-Ming G, Yong-Qing X, Yu-Shan W, Si-Miao T, Fan Y, Nan W, Shi-Bo H, Hui X, Xiao-Wei W, Hai-Shen J, Yu-Qiang Z, Jun A, Yuan-Liang C, Guang-Hua L, Yu-Jin L, Geng T, Zong-Sheng L, Yong C, Li M (2015) Prevalence of Nontraumatic Osteonecrosis of the Femoral Head and its Associated Risk Factors in the Chinese Population: Results from a Nationally Representative Survey. Chinese medical journal 128 
17. Daniel, Petek, Didier, Hannouche, Domizio, Suva (2019) Osteonecrosis of the femoral head: pathophysiology and current concepts of treatment. Efort Open Reviews

18. ShenYao Z, Fan W, XiangJing Z, Zhen H, KeFang D (2021) Astragalus polysaccharide ameliorates steroid-induced osteonecrosis of femoral head through miR-206/HIF-1a/BNIP3 axis. The Kaohsiung journal of medical sciences

19. Xu K, Lu C, Ren X, Wang J, Xu P, Zhang Y Overexpression of HIF-1a enhances the protective effect of mitophagy on steroid-induced osteocytes apoptosis. Environmental Toxicology

20. Zhipeng H, Qinglong W, Tao Z, Yinsheng F, Wenbo W (2021) Hyper-activated platelet lysates prevent glucocorticoid-associated femoral head necrosis by regulating autophagy. Biomedicine \& pharmacotherapy $=$ Biomedecine $\&$ pharmacotherapie 139

21. Yoon-Seok Y, Soo-Youn L, Soo-Ho L (2010) Apoptosis in the Osteonecrosis of the Femoral Head. Clin Orthop Surg 2:250-255

22. Xia X, Kar R, Gluhak-Heinrich J, Wei Y, Lane NE, Bonewald LF, Biswas SK, Lo WK, Jiang JX (2010) Glucocorticoid-Induced Autophagy in Osteocytes. Journal of Bone \& Mineral Research 25:24792488

23. Xing, Yin, Chenchen, Zhou, Jingtao, Li, Renkai, Liu, Bing, Shi (2019) Autophagy in bone homeostasis and the onset of osteoporosis. Bone Research v.7:16-31

24. Xudong Y, Shengnan Y, Xingzhi J, Jiachao G, Kai S, Fengjing G, Yaping Y (2020) PTEN inhibitor VOOHpic attenuates GC-associated endothelial progenitor cell dysfunction and osteonecrosis of the femoral head via activating Nrf2 signaling and inhibiting mitochondrial apoptosis pathway. Stem

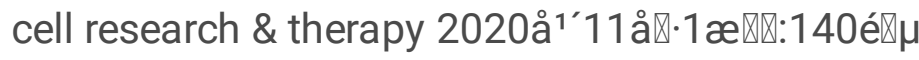

25. Zhang H, Zhou F, Pan Z, Bu X, Wang Y, Chen F (2017) 11ß-hydroxysteroid dehydrogenases-2 decreases the apoptosis of MC3T3/MLO-Y4 cells induced by glucocorticoids. Biochem Biophys Res Commun 490:1399

26. Demontis F, Perrimon N (2010) FOXO/4E-BP signaling in Drosophila muscles regulates organismwide proteostasis during aging. Cell 143:813-825

27. Wu X, Zhang Y, Guo X, Xu H, Xu Z, Duan D, Wang K (2015) Identification of differentially expressed microRNAs involved in non-traumatic osteonecrosis through microRNA expression profiling. Gene 565:22-29

28. Jia J, Yao W, Guan M, Dai W, Shahnazari M, Kar R, Bonewald L, Jiang JX, Lane NE (2011) Glucocorticoid dose determines osteocyte cell fate. Faseb Journal Official Publication of the Federation of American Societies for Experimental Biology 25:3366-3376

29. Korurer EG, Arga KY (2017) Differential co-expression analysis reveals a novel prognostic gene module in ovarian cancer. Scientific Reports 7:4996

30. Moriishi T, Fukuyama R, Miyazaki T, Furuichi T, Ito M, Komori T (2016) Overexpression of BCLXL in Osteoblasts Inhibits Osteoblast Apoptosis and Increases Bone Volume and Strength. Journal of Bone \& Mineral Research 31 
31. Huang B, Yu H, Li Y, Zhang W, Liu X (2019) Upregulation of long noncoding TNFSF10 contributes to osteoarthritis progression through the miR6/FGFR1 axis. Journal of Cellular Biochemistry 120

32. Xu Z, Yang H, Zhou X, Li J, Xu N (2019) Genetic variants in mTOR-pathway-related genes contribute to osteoarthritis susceptibility. International Immunopharmacology 77:105960

\section{Figures}
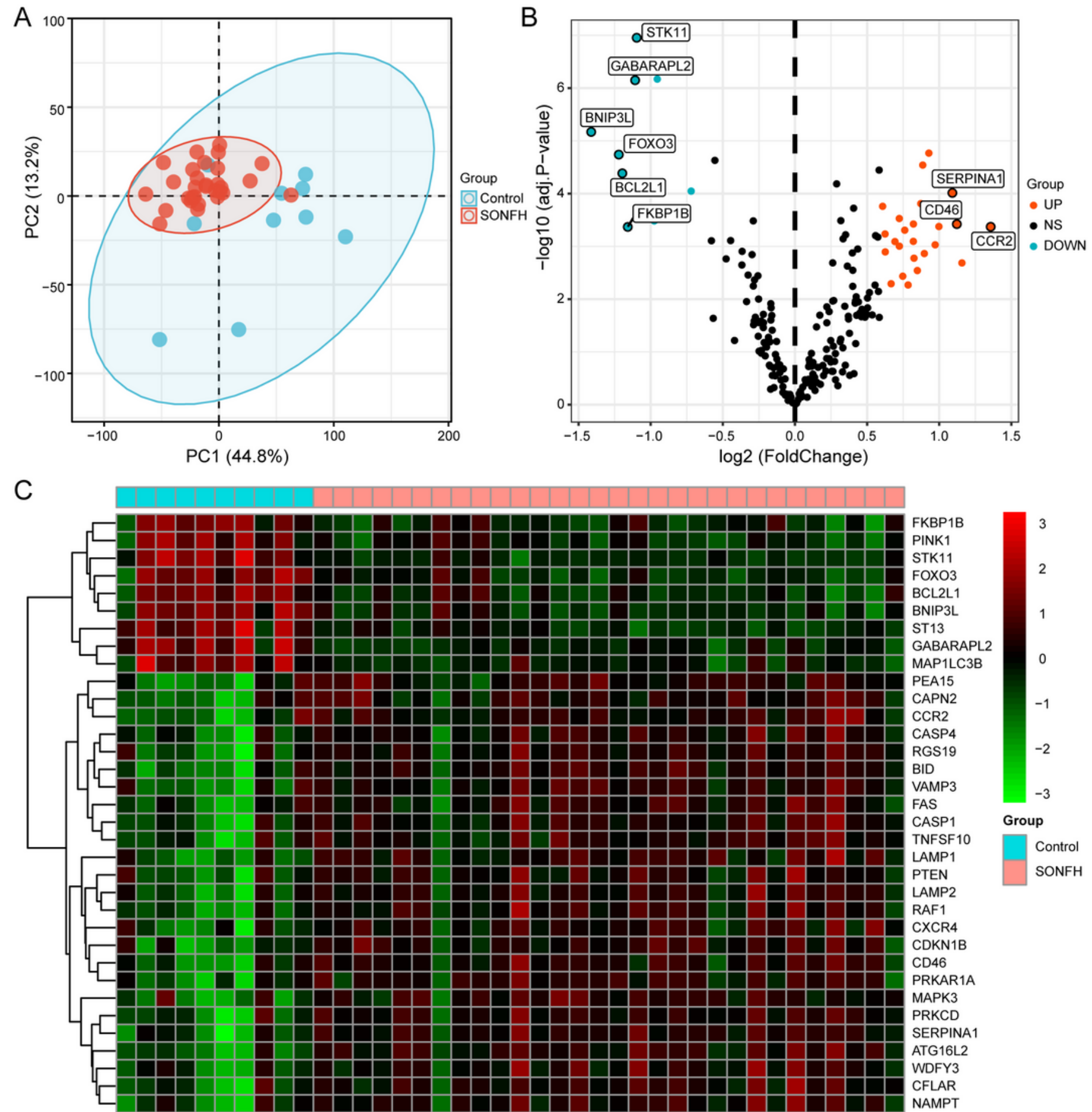

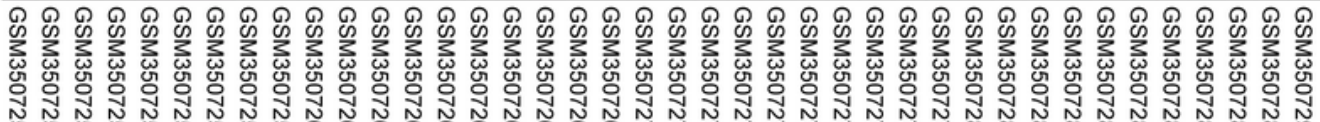

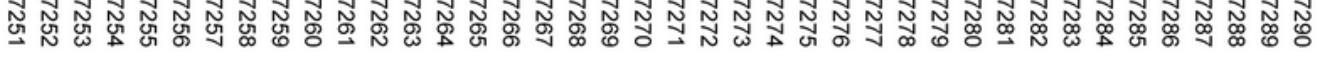


Figure 1

Differentially expressed autophagy-related genes in SONFH and non-SONFH samples. (A) Principal component analysis for GSE123568. (B) Volcano plot of the 222 differentially expressed autophagyrelated genes. The red dots represent the significantly up-regulated genes and the blue dots indicate the significantly down-regulated genes. (C) Hierarchical clustering heatmap of the 34 differentially expressed autophagy-related genes in SONFH and non-SONFH samples.

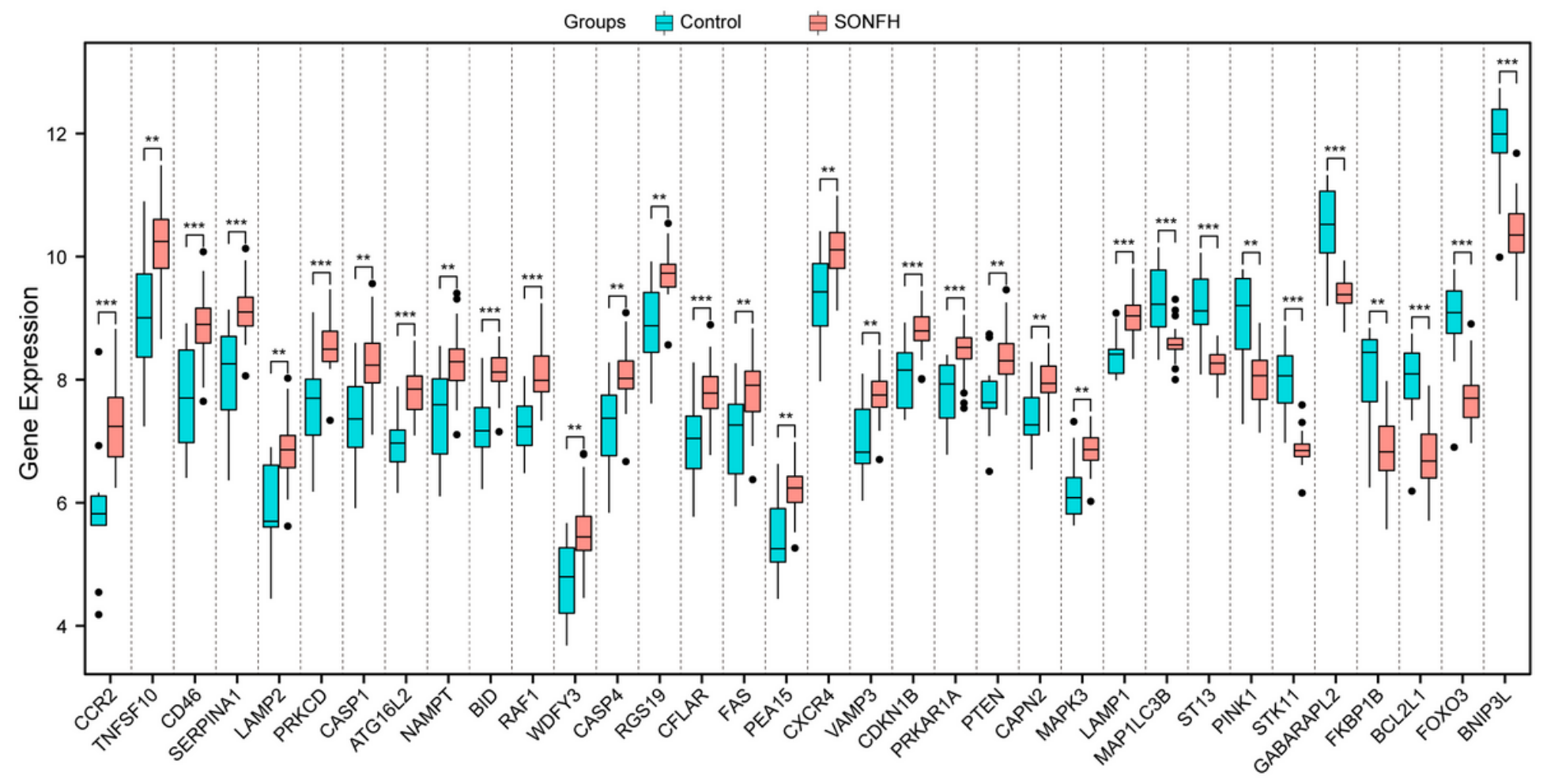

Figure 2

The boxplot of 34 differentially expressed autophagy-related genes in SONFH and non-SONFH samples. 
A

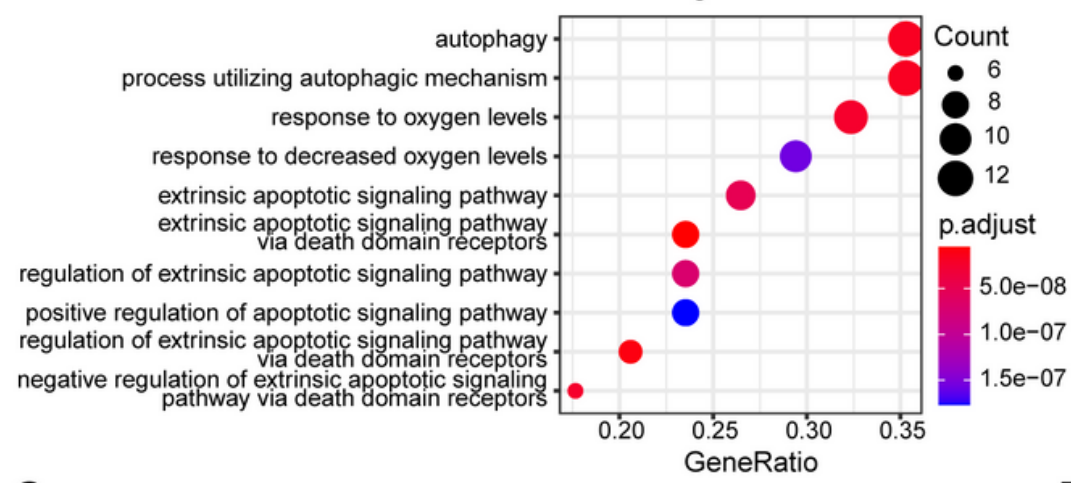

C

Molecular Function

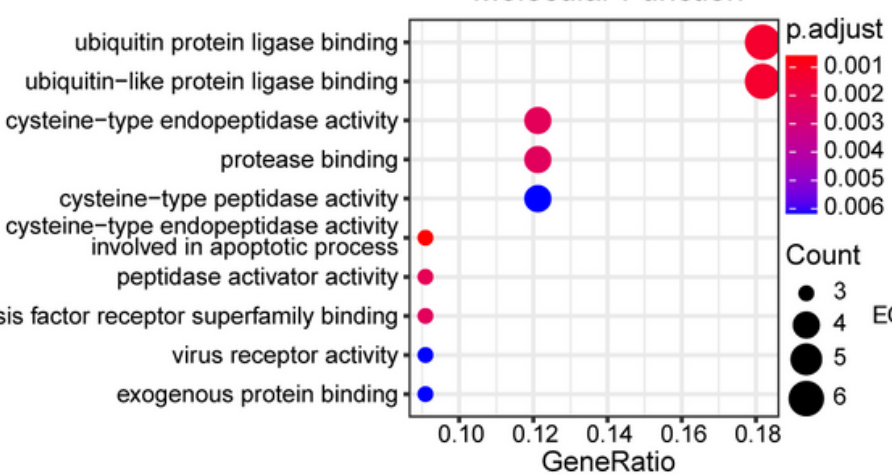

B

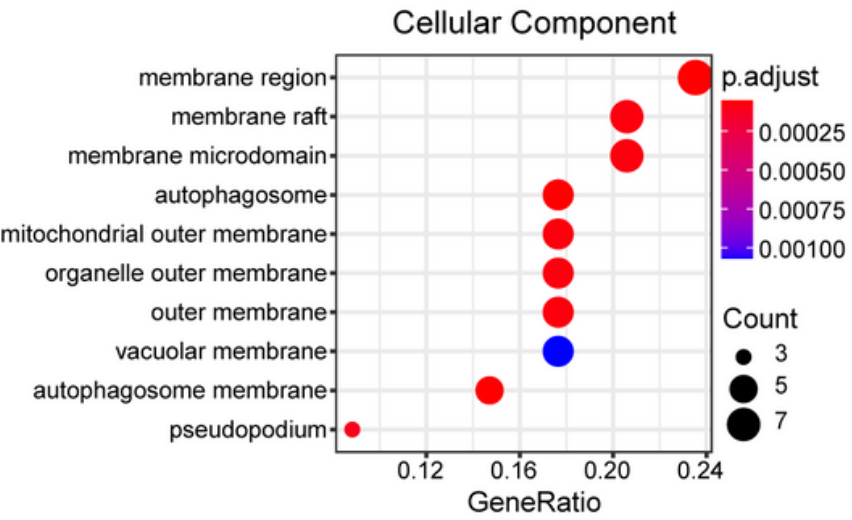

D

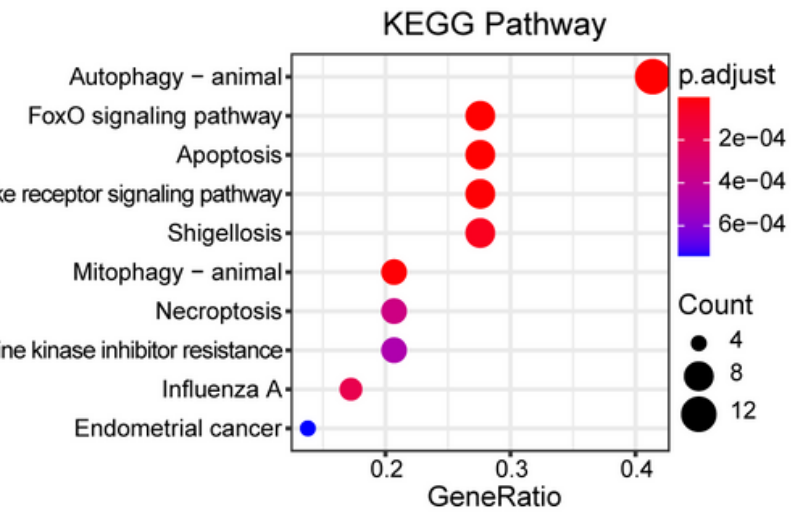

\section{Figure 3}

GO function enrichment analysis and KEGG pathway of 34 differentially expressed autophagy-related genes in SONFH and non-SONFH samples. (A) The bubble plot of top 10 enriched BP terms of 34 targets. (B) The bubble plot of top 10 enriched CC terms of 34 targets. (C) The bubble plot of top 10 enriched BP terms, CC terms and MF terms of 34 targets. (D) The bubble plot of top 10 enriched KEGG terms of 34 targets 


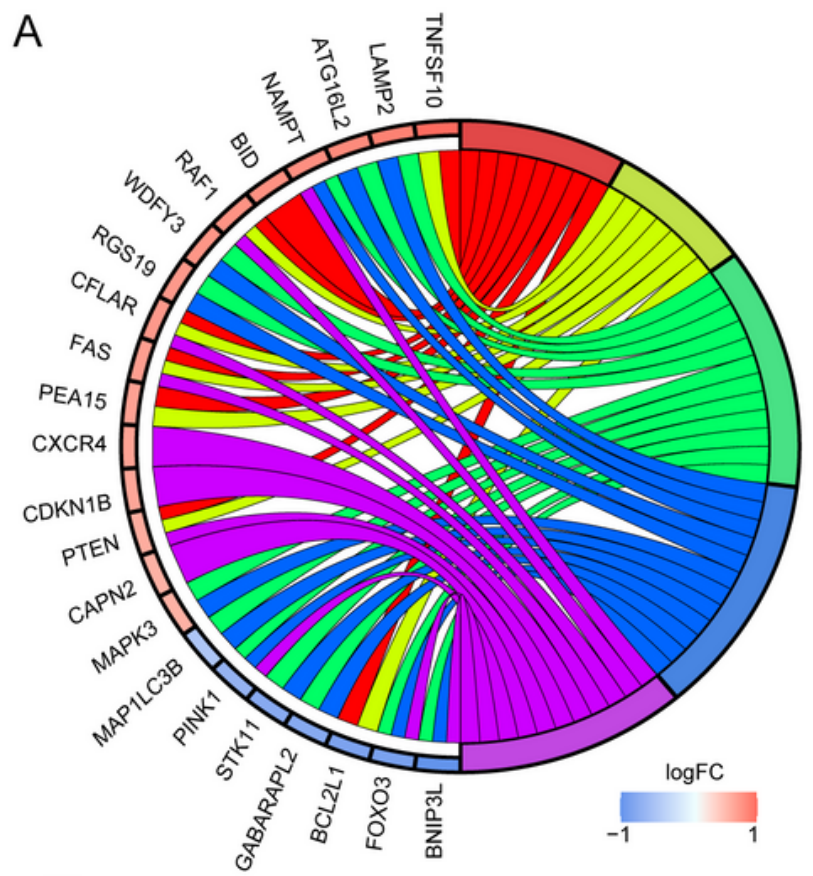

$\square$ extrinsic apoptotic signaling pathway via death domain receptors $\square$ regulation of extrinsic apoptotic signaling pathway via death domain receptors $\square$ autophagy

$\square$ process utilizing autophagic mechanism $\square$ response to oxygen levels

C

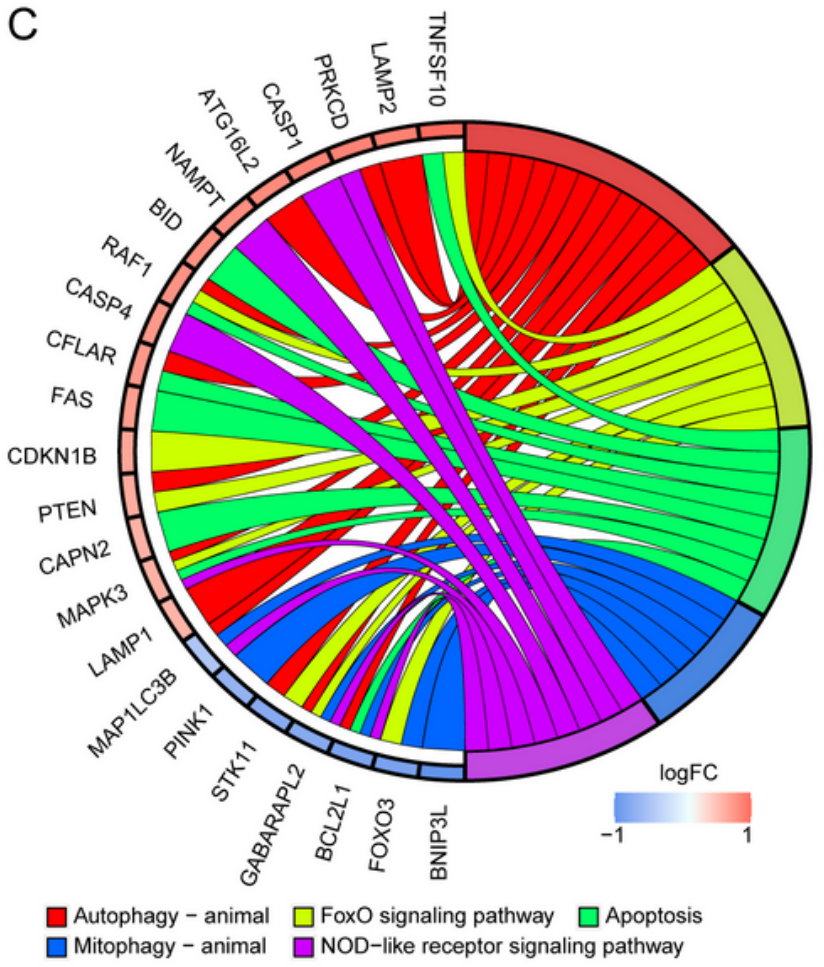

B

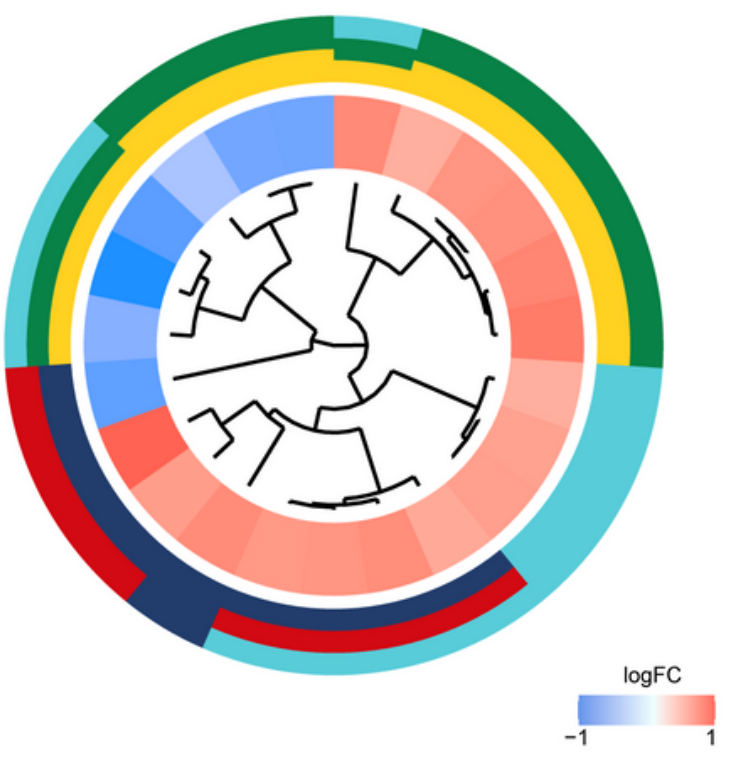

$\square$ extrinsic apoptotic signaling pathway via death domain receptors

$\square$ regulation of extrinsic apoptotic signaling pathway via death domain receptors $\square$ autophagy

$\square$ process utilizing autophagic mechanism

$\square$ response to oxygen levels

D

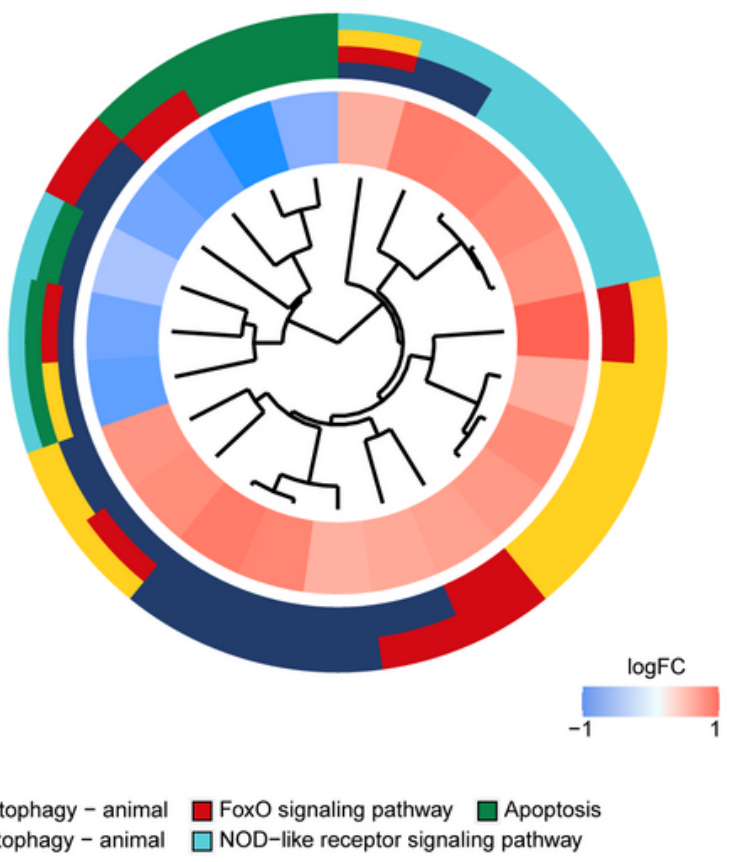

\section{Figure 4}

GO function enrichment analysis and KEGG pathway of 34 differentially expressed autophagy-related genes in SONFH and non-SONFH samples. (A) The relationship between top 5 enriched BP terms and targets was represented by the chord plot. (B) The relationship between top 5 enriched BP terms and targets was represented by the cluster plot. (C) The relationship between top 5 enriched KEGG pathway terms and targets was represented by the chord plot. (B) The relationship between top 5 enriched KEGG 
pathway terms and targets was represented by the cluster plot. The colors of the nodes were illustrated from red to blue in descending order of logFC. The genes were ordered according to logFC.

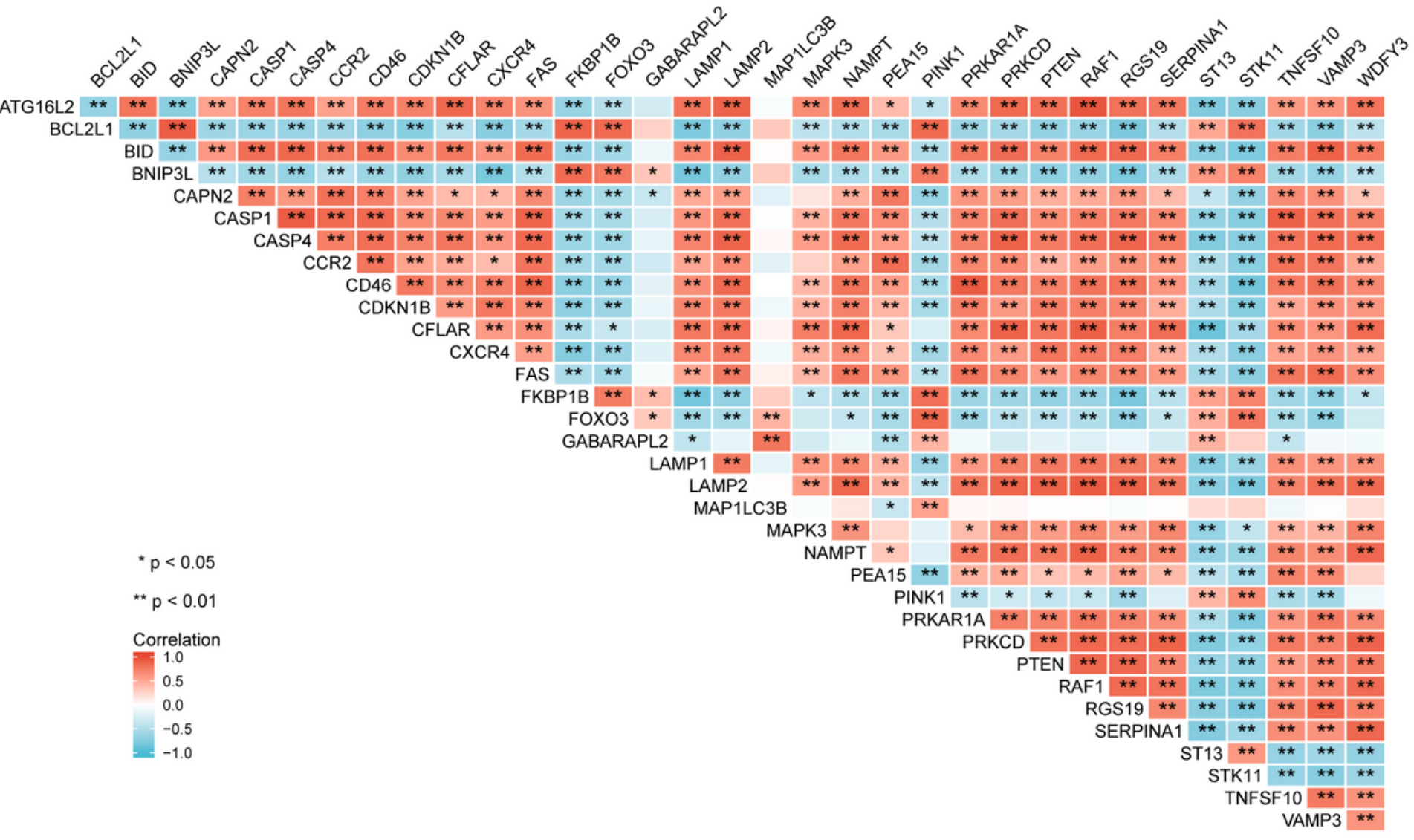

\section{Figure 5}

Spearman correlation analysis among the 34 differentially expressed autophagy-related genes in SONFH and non-SONFH samples.

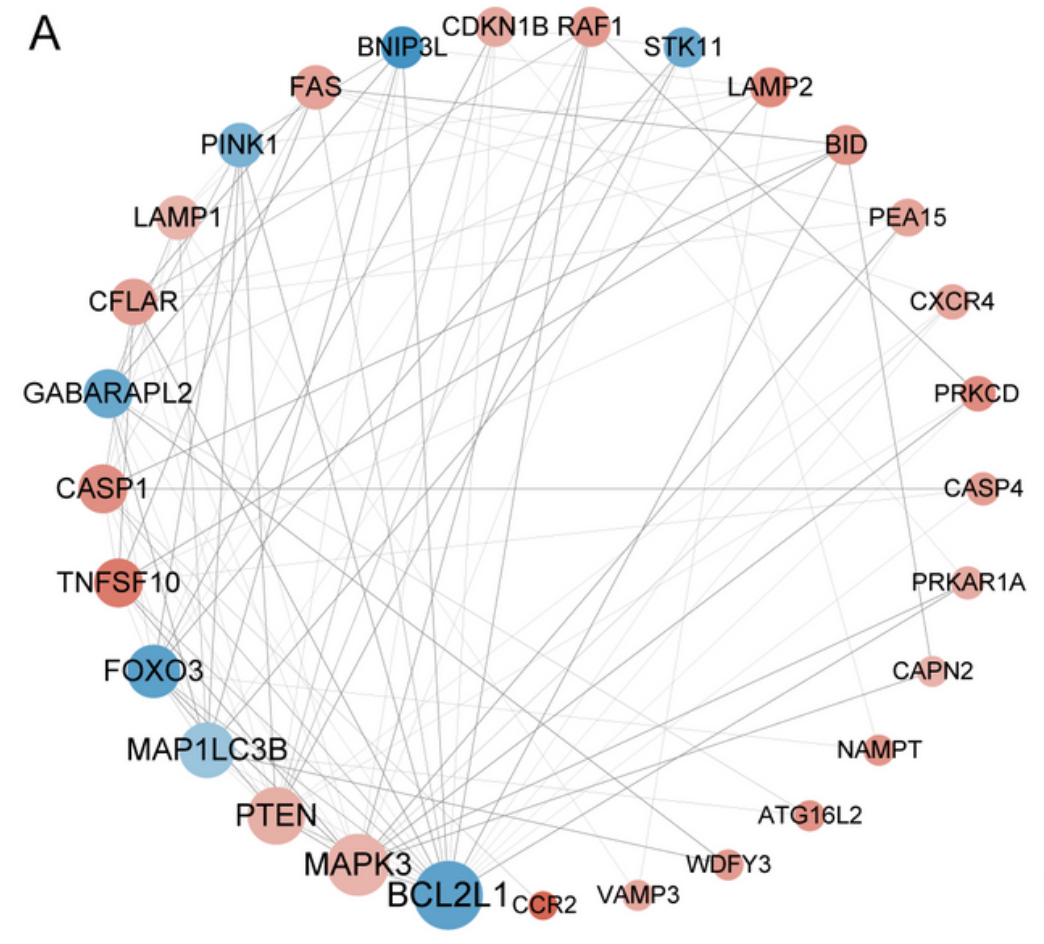

B

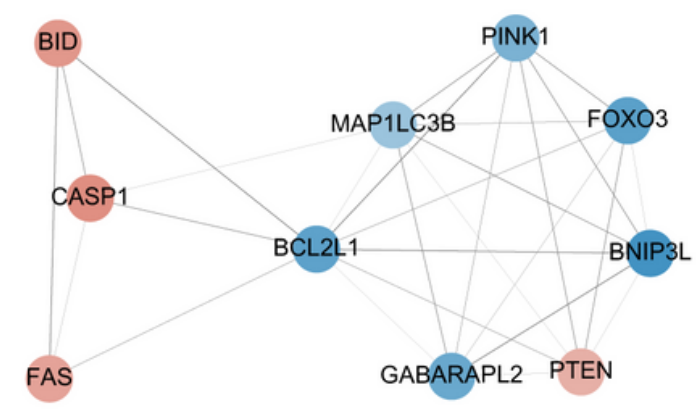

K-Core: 6.22210 nodes and 28 edges
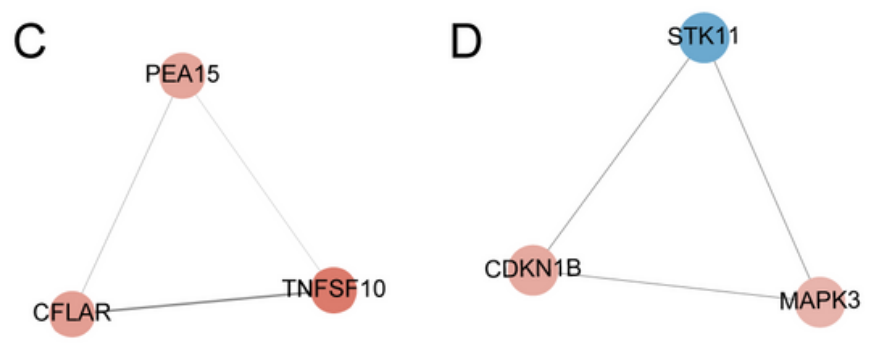

K-Core: 3.0003 nodes and 3 edges

K-Core: 3.0003 nodes and 3 edges 
Figure 6

PPI network of 34 differentially expressed autophagy-related genes and three cluster modules identified by MCODE in SONFH and non-SONFH samples. (A) The PPI network of differentially expressed autophagy-related genes in SONFH and non-SONFH samples was consisted of 29 nodes and 102 edges. Each node represented a protein, while each edge represented one protein-protein interaction. The color of node reflected the fold change, red color represented a higher upregulated gene, and blue color represented a lower downregulated gene; the size of node reflected the degree value, the larger the node, the greater the degree value. (B) Cluster 1: Score 6.222, 10 nodes and 28 edges; (C) Cluster 2: Score 3.000, 3 nodes and 3 edges; (D) Cluster 3: Score 3.000, 3 nodes and 3 edges.

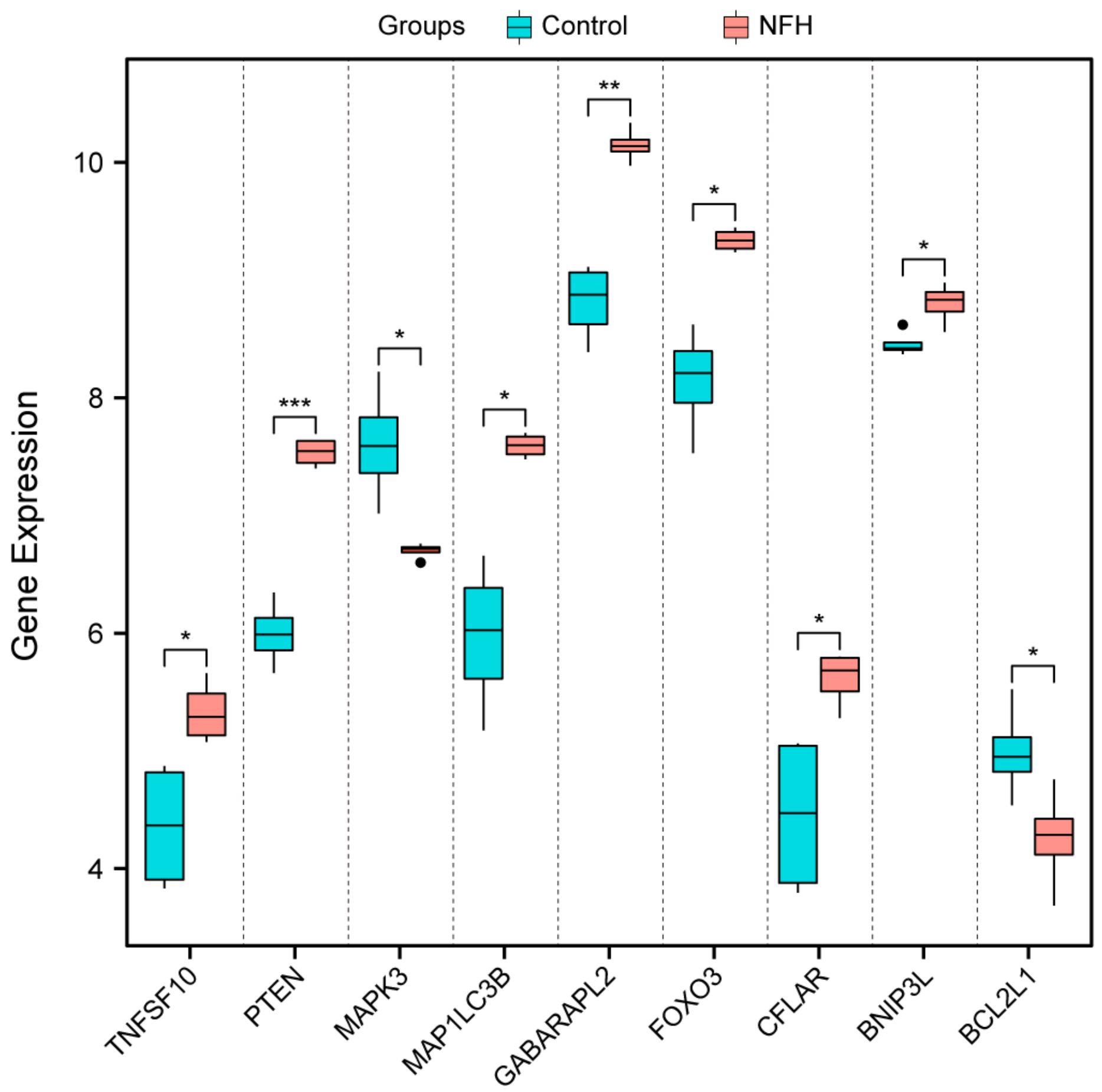




\section{Figure 7}

The boxplot of 10 differentially expressed autophagy-related genes in NFH and healthy samples of GSE74089 dataset. 